\title{
High-dose, short-interval daptomycin regimen was safe and well tolerated in three patients with chronic renal failure
}

This article was published in the following Dove Press journal:

Clinical Pharmacology:Advances and Applications

4 November 2013

Number of times this article has been viewed

\author{
Kaori Yabuno ${ }^{1,2, *}$ \\ Masafumi Seki ${ }^{1, *}$ \\ Koji Miyawaki ${ }^{1,2}$ \\ Yoshihiro Miwa² \\ Kazunori Tomono' \\ 'Division of Infection Control and \\ Prevention, ${ }^{2}$ Department of Pharmacy, \\ Osaka University Hospital, Osaka, \\ Japan \\ *These authors contributed equally \\ to this work
}

Background: The recommended daptomycin dosage is 4 or $6 \mathrm{mg} / \mathrm{kg} /$ day for the treatment of complicated skin and soft tissue infections or for Staphylococcus aureus bacteremia, endocarditis, and osteomyelitis. Every other day administration is usually recommended for patients with mild to moderate renal impairment. Higher doses ( $>6 \mathrm{mg} / \mathrm{kg} /$ day) have been explored as a possible alternative. Daptomycin is considered a safe anti-methicillin-resistant $S$. aureus (MRSA) drug, although renal dysfunction may be worsened. In this paper we report on three patients with chronic renal failure who received a higher dose of daptomycin daily for successful treatment for MRSA bacteremia, MRSA osteomyelitis, and methicillin-resistant S. epidermidis (MRSE) endocarditis.

Results: Previous administration of other drugs, including vancomycin, teicoplanin, and linezolid, had failed. In spite of daily treatment with daptomycin instead of the recommended alternate day regimen, adverse effects, such as elevation of creatinine and creatine phosphokinase, did not occur.

Conclusion: These experiences suggest that administration of high-dose/short-interval daptomycin can be efficient and safe even in the setting of renal dysfunction, and should be considered for the treatment of severe MRSA/MRSE infections in these patients.

Keywords: daptomycin, high-dose, renal failure, methicillin-resistant Staphylococcus aureus, creatine phosphokinase

\section{Introduction}

Daptomycin is a lipopeptide antibiotic with potent in vitro bactericidal activity against most clinically relevant strains of Gram-positive bacteria, including resistant strains such as methicillin-resistant Staphylococcus aureus (MRSA) and vancomycin-resistant enterococci. ${ }^{1,2}$ Recently, daptomycin has come to be used in Japan, as it is in more than 70 other countries. ${ }^{3,4}$

Currently, daptomycin is indicated for the treatment of complicated skin and soft tissue infections caused by susceptible organisms, as well as $S$. aureus bloodstream infections, including right-sided endocarditis., ${ }^{3,4}$ Unfortunately, clinical failures and resistant strains have already been reported for daptomycin. ${ }^{3,5}$ Consequently, doses greater than those approved are being considered as an alternative. ${ }^{3,4,6}$ Higher doses may be effective because of the pharmacokinetic profile of daptomycin and its concentrationdependent activity. ${ }^{3,7}$ Although clinical experience with doses $>6 \mathrm{mg} / \mathrm{kg} /$ day is limited, this dose has been increasingly utilized and daptomycin has been shown to be safe and well tolerated at doses up to $12 \mathrm{mg} / \mathrm{kg} /$ day in healthy volunteers. ${ }^{7}$ Nevertheless, use of high-dose daptomycin regimens remains controversial.
Correspondence: Masafumi Seki Division of Infection Control and Prevention, Osaka University Hospital, 2-I5 Yamadaoka, Suita,

Osaka 565-087I, Japan

Tel +8I 668795093

Fax +8I 668795094

Email seki@hp-infect.med.osaka-u.ac.jp 
Adverse events after daptomycin therapy have been reported, although the agent is generally well tolerated and safe. ${ }^{3}$ The tissue most sensitive to adverse events related to daptomycin is skeletal muscle, and some trials have demonstrated increased skeletal myopathy and elevation of creatine phosphokinase (CPK). ${ }^{3,6}$ Therefore, CPK values should be monitored weekly during daptomycin therapy and it is recommended that administration should be stopped in the event of CPK elevation.

Here, we present three patients with chronic renal failure who received a relatively high dose of daptomycin daily and experienced efficacy, both clinically and microbiologically. These patients experienced no adverse effects, including no elevation of CPK and creatinine, in spite of the fact that their estimated glomerular filtration rate was $10-30 \mathrm{~mL} / \mathrm{min} / 1.73 \mathrm{~m}^{2}$, for which setting the usual recommendation is administration every other day. ${ }^{8}$

\section{Case reports \\ Case I}

An 80-year-old woman who was diagnosed with infectious endocarditis due to methicillin-resistant Staphylococcus epidermis (MRSE) was administered daptomycin $5.9 \mathrm{mg} / \mathrm{kg}$ every other day, which is the regimen recommended in Japan (Figure 1). Three days later, daptomycin was changed to linezolid because inflammation markers had not improved; however, on day 7, nausea developed, suggesting an adverse effect of linezolid, so teicoplanin was started. Four days later, improvement in inflammation markers, such as numbers of white blood cells and C-reactive protein level, was less than expected, and nausea continued.

Therefore, we were consulted as infectious disease physicians; we suggested reinstituting daptomycin, but on a daily basis under careful observation, given that the every other day regimen had been ineffective on the first 3 days. Additional anti-MRSA drugs, such as rifampicin, were also considered, but the patient had a history of allergy to rifampicin. Unfortunately, mild interstitial pneumonia was subsequently seen on chest X-ray; however, the more expected adverse effects, such as elevation of CPK and creatinine, did not occur during the course of daptomycin treatment. The pneumonia improved with corticosteroid therapy soon after daptomycin administration was discontinued.

\section{Case 2}

A 75-year-old woman admitted to stabilize chronic heart failure was found to have MRSA bacteremia. Teicoplanin was started, but she complained of lumbago, and osteomyelitis was also found on magnetic resonance imaging. Therefore, treatment was changed to linezolid because better tissue penetration was expected (Figure 2). However, platelets decreased to less than $1 \times 10^{5}$ cells $/ \mu \mathrm{L}\left(4.3 \times 10^{4}\right)$ as an adverse effect of linezolid, and so we were consulted on day 28 . Because of the results from the patient described in case 1, who did not show an increase of CPK and creatinine, we suggested a change to daptomycin $6.2 \mathrm{mg} / \mathrm{kg}$ daily under close observation, in spite of her renal function (creatinine clearance $19.1 \mathrm{~mL}$ per minute). One month later, no adverse effects, including no elevation of CPK and creatinine, had occurred, and daptomycin therapy was clinically efficacious.

\section{Case 3}

An 85-year-old man who had undergone surgery for urinary bladder tumors was admitted because urinary infection was suspected. There were no findings indicating pyelonephritis by ultrasound examination, but meropenem $0.5 \mathrm{~g}$ twice daily

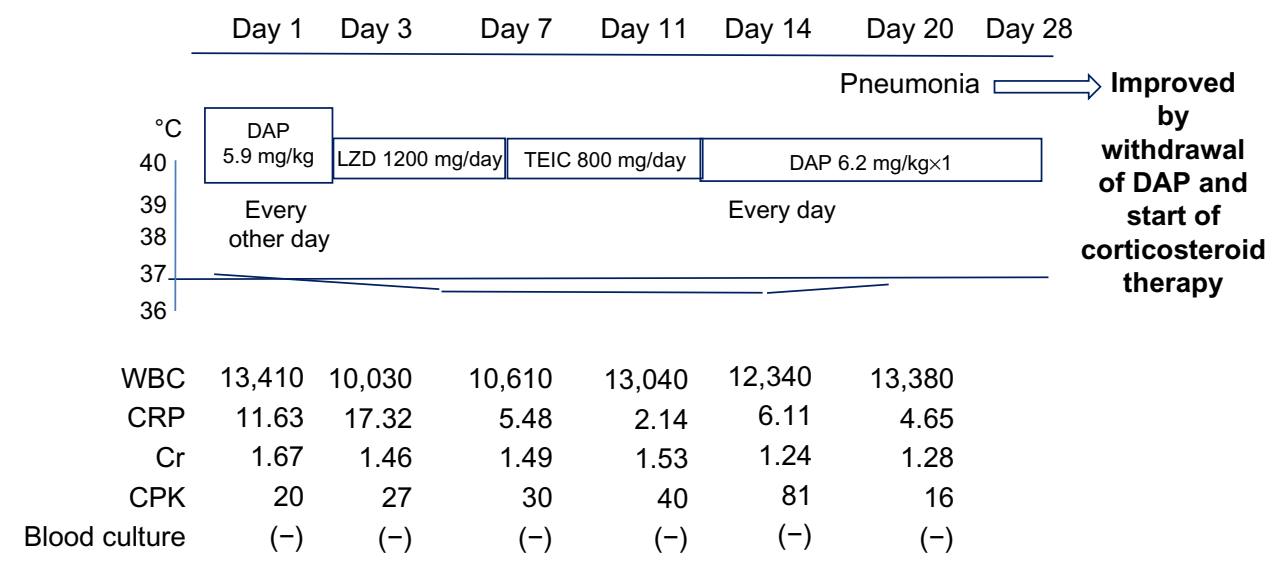

Figure I Clinical course of case I, an 80-year-old female who was treated for infectious endocarditis due to methicillin-resistant coagulase-negative Staphylococcus. Renal function: creatinine clearance $23.1 \mathrm{~mL}$ per minute and estimated glomerular filtration rate $20.3 \mathrm{~mL} / \mathrm{min} / \mathrm{I} .73 \mathrm{~m}^{2}$.

Abbreviations: WBC, white blood cells; CRP, C-reactive protein; Cr, creatinine; CPK, creatine phosphokinase; TEIC, teicoplanin; DAP, daptomycin; LZD, linezolid. 


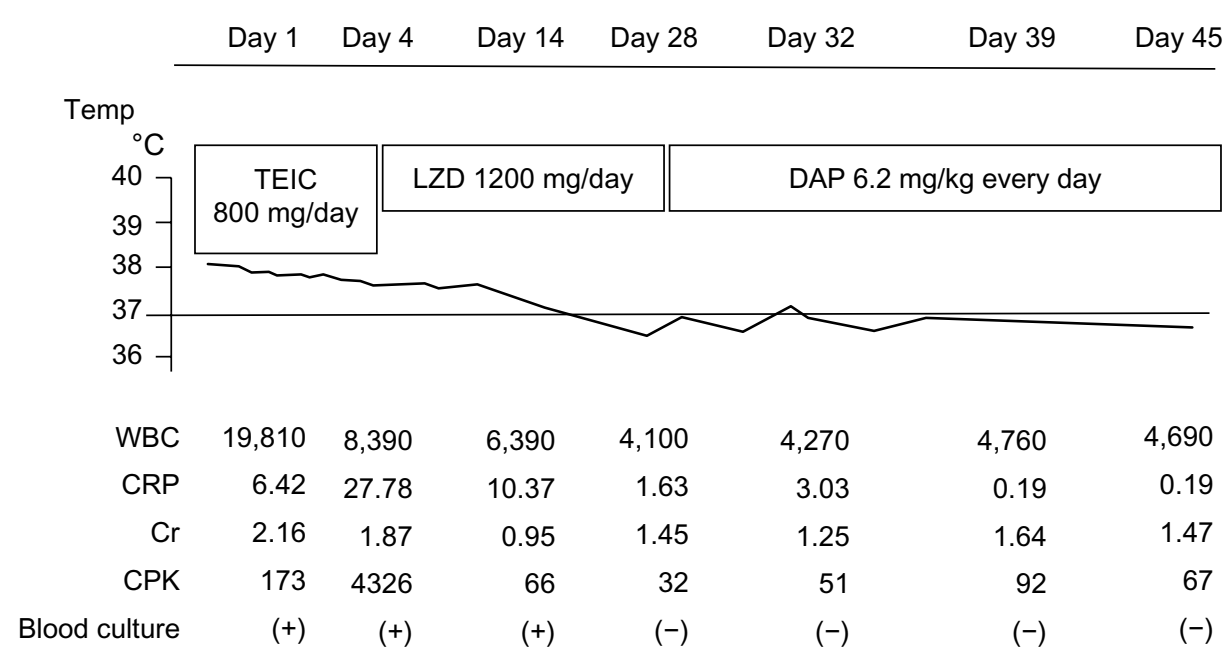

Platelet $43,000 \mathrm{cells} / / \mathrm{mL}$

Figure 2 Clinical course of case 2, a 75-year-old female who was treated for methicillin-resistant Staphylococcus aureus osteomyelitis. Renal function: creatinine clearance $19.1 \mathrm{~mL}$ per minute and estimated glomerular filtration rate $20.1 \mathrm{~mL} / \mathrm{min} / 1.73 \mathrm{~m}^{2}$.

Abbreviations: WBC, white blood cells; CRP, C-reactive protein; $\mathrm{Cr}$, creatinine; CPK, creatine phosphokinase; TEIC, teicoplanin; DAP, daptomycin; LZD, linezolid.

was begun empirically by the primary physicians because of their suspicion of severe sepsis due to Gram-negative rods, including extended-spectrum beta-lactamase-producing strains of Escherichia coli and Klebsiella species, which are seen (about $>20 \%$ ) in urology patients in Japan (Figure 3). However, 2 days later, MRSA, with a minimum inhibitory concentration for vancomycin of $1 \mu \mathrm{g} / \mathrm{mL}$ (susceptible), was isolated from the blood culture, and vancomycin was started at a dose determined by the renal function (creatinine clearance $16.3 \mathrm{~mL}$ per minute). The dosage of meropenem and vancomycin determined by the primary physicians was quite low at first (half of the usual) because of concern for possible deterioration of renal function. In fact, vancomycin was ineffective and the trough level was quite low $(7.8 \mu \mathrm{g} / \mathrm{mL})$. Further, MRSA was again isolated from the day 3 blood culture, and inflammation indicators, such as white blood cells and C-reactive protein level, had not improved. Indicators of the physical condition of the patient, such as blood pressure and respiratory status, had also worsened; therefore, we were consulted and, because of our experiences with cases 1 and 2, we again suggested that vancomycin should be changed to daptomycin $8 \mathrm{mg} / \mathrm{kg}$ daily under

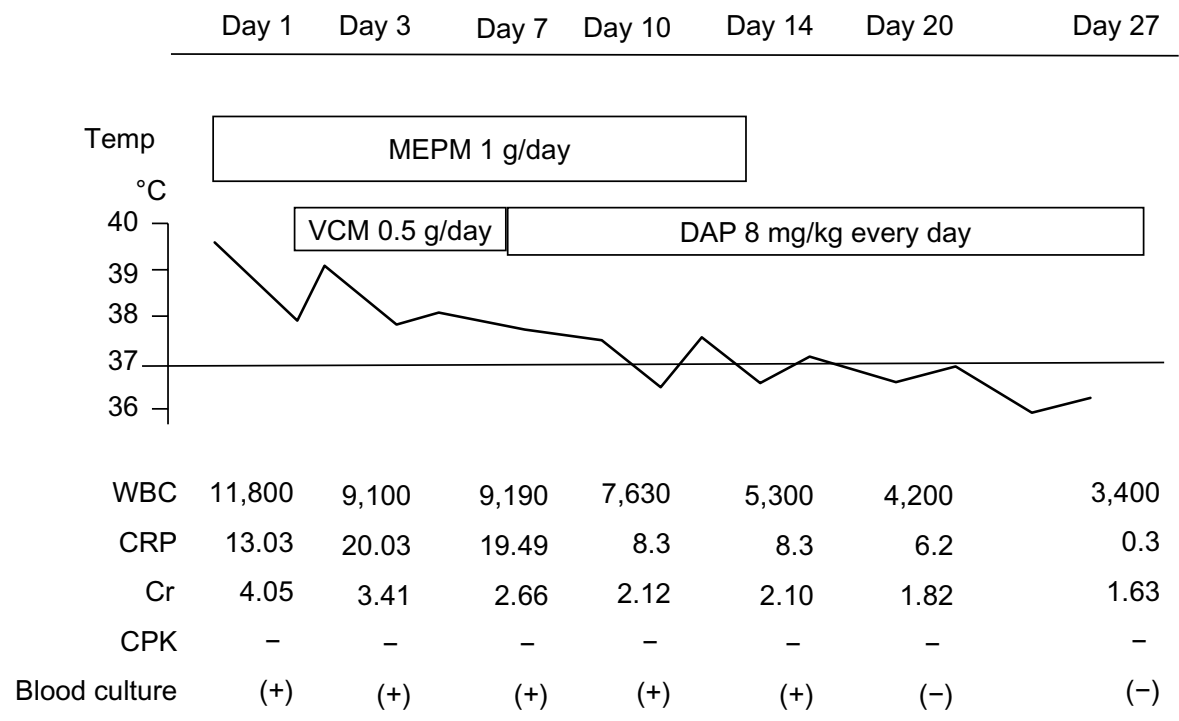

Figure 3 Clinical course of case 3, an 85-year-old male who was treated for methicillin-resistant Staphylococcus aureus bacteremia. Renal function: creatinine clearance $16.3 \mathrm{~mL}$ per minute and estimated glomerular filtration rate $10.2 \mathrm{~mL} / \mathrm{min} / 1.73 \mathrm{~m}^{2}$.

Abbreviations: WBC, white blood cells; CRP, C-reactive protein; Cr, creatinine; CPK, creatine phosphokinase; MEPM, meropenem; VCM, vancomycin; DAP, daptomycin. 
careful observation. After administration of daptomycin, blood cultures became negative for MRSA, and inflammation markers also improved. No adverse effects were noted, including no decreased creatinine clearance.

\section{Discussion}

Daptomycin is an antimicrobial agent used to treat Grampositive organisms, including MRSA, vancomycin-resistant enterococci, and MRSE, ${ }^{1,4}$ It has been successfully used in the treatment of complicated infections due to these Grampositive multidrug-resistant pathogens, especially endocarditis, wound infections, and device-related and catheter-related infections in intensive care units.

In this report, we present three patients successfully treated with a relatively high-dose, short-interval (daily) daptomycin regimen, instead of the more usual lower-dose, every other day regimen. A retrospective chart review has been reported for all patients treated with high-dose daptomycin from 2004 to $2007 .{ }^{9}$ The investigators reported that $22(36.1 \%)$ of 61 patients experienced mild adverse events (eg, diarrhea, nausea, and arthralgia) that did not necessitate discontinuation of daptomycin. In 47 patients who had CPK analysis (either paired at the beginning of and during therapy or at random during treatment), significant CPK elevation was observed in only three cases (6.4\%). Thus, it was concluded that high-dose daptomycin at a mean dose of $8 \mathrm{mg} / \mathrm{kg} /$ day was well tolerated. Consistent with that report, elevation of CPK did not occur in the three current cases; our experience also supports the suggestion that higher and continuous use of daptomycin might be acceptable, rather than the recommended 4-6 $\mathrm{mg} / \mathrm{kg}$ every other day regimen, which was safe but sometimes less effective, as it was initially for the current case $1 .^{3}$

Moise et al analyzed 106 patients who received daptomycin for $S$. aureus bacteremia and had mild (creatinine clearance $30-50 \mathrm{~mL}$ per minute) or moderate (creatinine clearance 10-30 mL per minute, no dialysis) renal insufficiency, and found that daptomycin treatment was successful in $81 \%$ of patients. ${ }^{10}$ In that study, the treatment response differed significantly $(P=0.006)$ according to the degree of renal insufficiency, with higher failure rates occurring among those with moderate renal insufficiency receiving the usual regimen. Only two patients experienced an increase in CPK, which resolved after stopping daptomycin. No patients developed worsening renal insufficiency related to daptomycin administration.

In our three cases, the decreased renal function might have been due to sepsis, but treatment with high-dose daily daptomycin was not only effective but also did not increase creatinine and CPK. Recently, it has been suggested that unsuccessful treatment of mild to moderate renal failure patients using daptomycin may be because of use of lower doses and/or long intervals between doses. ${ }^{10}$ Our experience with these cases suggests that a higher-dose, shorter-interval regimen than is usually recommended today is effective for patients with renal impairment.

The European Cubicin Outcomes Registry and Experience (EU-CORE) study of 1,127 patients reported that there was no increase in the proportion of patients with creatinine clearance $<30 \mathrm{~mL}$ per minute after initiation of daptomycin treatment. ${ }^{18}$ In the EU-CORE study, elevated CPK was reported as a severe adverse effect in only one patient. However, three patients in a report from New York developed serum CPK elevations $>1,000 \mathrm{U} / \mathrm{L}$ and all were symptomatic with complaints of constitutional and/or musculoskeletal symptoms following 24-28 days of daptomycin treatment. ${ }^{9}$ Therapy was discontinued in these three patients, leading to prompt resolution of symptoms and the CPK abnormality. Therefore, administration of daptomycin could be withdrawn when any abnormal laboratory data and symptoms occur; in special circumstances, use of the higher-dose daptomycin regimen under careful observation, including daily blood collection for laboratory analysis, may be appropriate. The current three patients were carefully observed by nephrologists and urologists.

In the current case 1, mild pneumonia occurred and might have been due to administration of daptomycin; this improved after withdrawal of daptomycin and initiation of steroid therapy. Although more than one million patients worldwide received daptomycin in 2004-2010, only seven cases of definitive eosinophilic pneumonia related to daptomycin were confirmed. ${ }^{11}$ All seven cases improved or were cured after suspension of daptomycin therapy, and five were treated with corticosteroids. Drug-induced pneumonia as a consequence of daptomycin therapy is very rare, and is often treatable. However, a change to another anti-MRSA drug was needed for the two other reported cases who developed recurrent pneumonia after readministration of daptomycin.

Multiple cases of treatment with high-dose daptomycin in patients with normal renal function have been reported in the literature, and the investigators have attributed clinical success to the increased daptomycin dosage. ${ }^{3,12-14}$ Dosages of $8-12 \mathrm{mg} / \mathrm{kg}$ daily have been utilized for prolonged periods of time for the treatment of acute bacterial MRSA endocarditis, MRSA bacteremia, vancomycin-resistant enterococcal 
endocarditis, and prosthetic infections. We have also used these high-dose daptomycin regimens for patients with normal renal function: only $1 / 12(8.3 \%)$ patients had elevation of CPK, and treatment was clinically effective in $8 / 12$ (66.7\%, data not shown). We did not adequately analyze the efficiency of high-dose daptomycin by comparing with cases who received regular or lower doses; further detailed study will be needed to confirm the efficiency and safety of the high-dose daptomycin regimen.

An additional problem to anticipate is the possibility of increased resistance to daptomycin if higher dosages are used as regular treatment in the future. Emergence of daptomycin resistance in MRSA has been reported. ${ }^{15}$ Wild-type $S$. aureus with minimum inhibitory concentrations of daptomycin greater than the susceptible range might be rare, but had been recovered from patients who received vancomycin, patients who had never received daptomycin, and patients who were antibiotic-naïve. ${ }^{16,17}$ Spontaneous resistance is uncommon, but resistance can be induced in vitro by serial passage in increasing concentrations of daptomycin. ${ }^{18}$ We need to remain vigilant against the appearance of daptomycin-resistant MRSA when using high-dose regimens.

In conclusion, we treated three patients who tolerated relatively high-dose daily daptomycin administration in spite of renal dysfunction, a condition for which an every other day regimen is usually recommended. There was no elevation of CPK or creatinine, and there was improvement of their bacteremia, endocarditis, and osteomyelitis. Other anti-MRSA drugs and daptomycin every other day were not as effective in these cases. We suggest, therefore, that a more continuous and higher-dose regimen of daptomycin should be considered as initial therapy for very severe infections, even in the setting of moderate renal insufficiency. These patients should be carefully monitored by laboratory examinations for early detection of any deterioration of renal function. Use of this high-dose regimen must be confirmed in a larger number of patients with renal failure.

\section{Disclosure}

The authors declare that they have no conflicts of interest regarding the publication of this paper.

\section{References}

1. Tedesco KL, Rybak MJ. Daptomycin. Pharmacotherapy. 2004;24: $41-57$.
2. Dvorchik BH, Brazier D, DeBruin MF, Arbeit RD. Daptomycin pharmacokinetics and safety following administration of escalating doses once daily to healthy subjects. Antimicrob Agents Chemother. 2003;47: $1318-1323$.

3. Wu G, Abraham T, Rapp J, Vastey F, Saad N, Balmir E. Daptomycin: evaluation of a high-dose treatment strategy. Int J Antimicrob Agents. 2011;38:192-196.

4. Liu C, Bayer A, Cosgrove SE, et al. Clinical practice guidelines by the infectious diseases society of america for the treatment of methicillinresistant Staphylococcus aureus infections in adults and children. Clin Infect Dis. 2011;52:e18-e55.

5. Julian K, Kosowska-Shick K, Whitener C, et al. Characterization of a daptomycin-nonsusceptible vancomycin-intermediate Staphylococcus aureus strain in a patient with endocarditis. Antimicrob Agents Chemother. 2007;51:3445-3448.

6. Moise PA, Hershberger E, Amodio-Groton MI, Lamp KC. Safety and clinical outcomes when utilizing high-dose $(>$ or $=8 \mathrm{mg} / \mathrm{kg}$ ) daptomycin therapy. Ann Pharmacother. 2009;43:1211-1219.

7. Benvenuto M, Benziger DP, Yankelev S, Vigliani G. Pharmacokinetics and tolerability of daptomycin at doses up to 12 milligrams per kilogram of body weight once daily in healthy volunteers. Antimicrob Agents Chemother. 2006;50:3245-3249.

8. Gonzalez-Ruiz A, Beiras-Fernandez A, Lehmkuhl H, Seaton RA, Loeffler J, Chaves RL. Clinical experience with daptomycin in Europe: the first 2.5 years. J Antimicrob Chemother. 2011;66:912-919.

9. Figueroa DA, Mangini E, Amodio-Groton M, et al. Safety of high-dose intravenous daptomycin treatment: three-year cumulative experience in a clinical program. Clin Infect Dis. 2009;49:177-180.

10. Moise PA, Amodio-Groton M, Rashid M, et al. Multicenter evaluation of the clinical outcomes of daptomycin with and without concomitant $\beta$-lactams in patients with Staphylococcus aureus bacteremia and mild to moderate renal impairment. Antimicrob Agents Chemother. 2013;57:1192-1200.

11. US Food and Drug Administration. Drug Safety Communication. Eosinophilic pneumonia associated with the use of Cubicin (daptomycin). 2010. Available from: http://www.fda.gov/Drugs/DrugSafety/ PostmarketDrugSafetyInformationforPatientsandProviders/ ucm220273.htm. Accessed October 4, 2013.

12. Lichterfeld M, Ferraro MJ, Davis BT. High-dose daptomycin for the treatment of endocarditis caused by Staphylococcus aureus with intermediate susceptibility to glycopeptides. Int J Antimicrob Agents. 2010;35:96

13. Cunha BA, Krol V, Kodali V. Methicillin-resistant Staphylococcus aureus (MRSA) mitral valve acute bacterial endocarditis (ABE) in a patient with Job's syndrome (hyperimmunoglobulin E syndrome) successfully treated with linezolid and high-dose daptomycin. Heart Lung. 2008;37:72-75.

14. Bassetti M, Nicco E, Ginocchio F, Ansaldi F, de Florentiis D, Viscoli C. High-dose daptomycin in documented Staphylococcus aureus infections. Int J Antimicrob Agents. 2010;36:459-461.

15. Boucher HW, Sakoulas G. Perspectives on daptomycin resistance, with emphasis on resistance in Staphylococcus aureus. Clin Infect Dis. 2007;45:601-608.

16. Pfaller MA, Sader H, Jones RN. Evaluation of the in vitro activity of daptomycin against 19,615 clinical isolates of gram-positive cocci collected in North American hospitals (2002-2005). Diagn Microbiol Infect Dis. 2007;57:459-465.

17. Jones RN, Stilwell MG, Sader HS, Fritsche TR, Goldstein BP. Spectrum potency of dalbavancin tested against $3322 \mathrm{Gram}$-positive cocci isolated in the United States Surveillance Program (2004). Diagn Microbiol Infect Dis. 2006;54:149-153.

18. Silverman JA, Oliver N, Andrew T, Li T. Resistance studies with daptomycin. Antimicrob Agents Chemother. 2001;45:1799-1802. 


\section{Publish your work in this journal}

Clinical Pharmacology: Advances and Applications is an international, Visit http://www.dovepress.com/testimonials.php to read real quotes peer-reviewed, open access journal publishing original research, reports,

reviews and commentaries on all areas of drug experience in humans.

The manuscript management system is completely online and includes

a very quick and fair peer-review system, which is all easy to use.

from published authors.

Submit your manuscript here: http://www.dovepress.com/clinical-pharmacology-advances-and-applications-journal 